\title{
Characterization and Enumeration of Complementary Dual Abelian Codes*
}

\author{
Arunwan Boripan ${ }^{\dagger}$, Somphong Jitman $\ddagger$ and Patanee Udomkavanich ${ }^{\S}$
}

April 23, 2022

\begin{abstract}
Abelian codes and complementary dual codes form important classes of linear codes that have been extensively studied due to their rich algebraic structures and wide applications. In this paper, a family of abelian codes with complementary dual in a group algebra $\mathbb{F}_{p^{\nu}}[G]$ has been studied under both the Euclidean and Hermitian inner products, where $p$ is a prime, $\nu$ is a positive integer, and $G$ is an arbitrary finite abelian group. Based on the discrete Fourier transform decomposition for semi-simple group algebras and properties of ideas in local group algebras, the characterization of such codes have been given. Subsequently, the number of complementary dual abelian codes in $\mathbb{F}_{p^{\nu}}[G]$ has been shown to be independent of the Sylow $p$-subgroup of $G$ and it has been completely determined for every finite abelian group $G$. In some cases, a simplified formula for the enumeration has been provided as well. The known results for cyclic complementary dual codes can be viewed as corollaries.
\end{abstract}

Keywords: Complementary dual codes, Abelian codes, Group algebras, Local group algebra

Mathematics Subject Classification: 94B15, 94B05, 16A26

\section{Introduction}

Abelian codes form an important class of linear codes that has been extensively studied for both theoretical and practical reasons (see, for example, [3], 6], [7], [12, 13], 20], and [21]). Moreover, this class of codes contains the classical cyclic codes which can be efficiently encoded and decoded using shift registers. Linear complementary dual (LCD) codes introduced in [14] constitute another interesting class of linear codes. It has been shown that LCD codes are asymptotically good and they can be suitably applied for the two-user binary adder channel in [14. In [22], it has been shown that LCD codes meet the Gilbert-Varshamov bound.

\footnotetext{
${ }^{*}$ This research was supported by the Thailand Research Fund under Research Grant MRG6080012.

${ }^{\dagger}$ A. Boripan is with the Department of Mathematics and Computer Science, Faculty of Science, Chulalongkorn University, Bangkok 10330, Thailand. boripan-arunwan@hotmail.com

${ }^{\ddagger}$ S. Jitman (Corresponding Author) is with the Department of Mathematics, Faculty of Science, Silpakorn University, Nakhon Pathom 73000, Thailand. sjitman@gmail.com

${ }^{\S} \mathrm{P}$. Udomkavanich is with the Department of Mathematics and Computer Science, Faculty of Science, Chulalongkorn University, Bangkok 10330, Thailand. pattanee.u@chula.ac.th
} 
LCD codes are also useful in information protection from side-channel attacks and hardware Trojan horses (see [4], [5], [8], [10], [16], and [17]). In [9], LCD codes have been applied in constructing good entanglement-assisted quantum error correcting codes. It is therefore of natural interest to study algebraic structured codes with complementary dual. Cyclic codes with complementary dual have been studied in [23]. Necessary and sufficient conditions for cyclic codes to be complementary dual with respect to the Euclidean inner product have been given in [23].

In this paper, we focus on a more general setup. Precisely, abelian codes with complementary dual are studied under both the Euclidean and Hermitian inner products. We focus on the characterization and enumeration of such codes. Based on the discrete Fourier transform decomposition for semi-simple group algebras, the group algebra $\mathbb{F}_{p^{\nu}}[G]$ can be view as a product of local group algebras for all finite abelian groups $G$. The complementary dual abelian codes and the direct summand ideals in each local component are characterized. The characterization and enumeration of complementary dual abelian codes can be obtained through the discrete Fourier transform decomposition and the characterization of ideals in local group algebras mentioned above. A simplified formula for the enumeration is given as well in the case where the underlying group is a cyclic group or a $q$-group, where $q$ is a prime such that $q \neq p$.

The paper is organized as follows. Basic properties of group algebras and abelian codes are recalled in Section 2. Complementary dual abelian codes in some local group algebras are studied in Section 3. Based on the results in Section 3, it is shown that the characterization and enumeration of Euclidean complementary dual abelian codes over finite fields of characteristics $p$ are independent of the Sylow $p$-subgroup of $G$. The complete characterization and enumeration of Euclidean complementary dual abelian codes are given in Section 4. The analogous results for Hermitian complementary dual abelian codes are given in Section 5 .

\section{Preliminaries}

Let $R$ be a commutative ring with identity and let $G$ be a finite abelian group, written additively. Denote by $R[G]$ the group ring of $G$ over $R$. The elements in $R[G]$ will be written as $\sum_{g \in G} \alpha_{g} Y^{g}$, where $\alpha_{g} \in R$. The addition and the multiplication in $R[G]$ are given as in the usual polynomial rings over $R$ with the indeterminate $Y$, where the indices are computed additively in $G$. The homomorphism $\epsilon: R[G] \rightarrow R$ defined by

$$
\sum_{g \in G} \alpha_{g} Y^{g} \mapsto \sum_{g \in G} \alpha_{g}
$$

is called an augmentation map and the kernel $\Delta_{R}(G):=\operatorname{ker}(\epsilon)$ is called an augmentation ideal of $R[G]$.

For a prime $p$ and a positive integer $\nu$, let $\mathbb{F}_{p^{\nu}}$ denote the finite field of order $p^{\nu}$. In the case where $R=\mathbb{F}_{p^{\nu}}$, the group ring $\mathbb{F}_{p^{\nu}}[G]$ is called a group algebra. An abelian code in $\mathbb{F}_{p^{\nu}}[G]$ is defined to be an ideal in $\mathbb{F}_{p^{\nu}}[G]$ (see [12] and [13]). The Euclidean inner product between $u=\sum_{g \in G} u_{g} Y^{g}$ and $v=\sum_{g \in G} v_{g} Y^{g}$ in $\mathbb{F}_{p^{\nu}}[G]$ is defined to be $\langle u, v\rangle_{\mathrm{E}}:=\sum_{g \in G} u_{g} v_{g}$. The Euclidean dual of an abelian code $C$ in $\mathbb{F}_{p^{\nu}}[G]$ is defined to be $C^{\perp_{\mathrm{E}}}:=\left\{v \in \mathbb{F}_{p^{\nu}}[G] \mid\langle c, v\rangle_{\mathrm{E}}=0\right.$ for all $\left.c \in C\right\}$. An abelian code $C$ 
in $\mathbb{F}_{p^{\nu}}[G]$ is said to be Euclidean complementary dual if $C \cap C^{\perp_{\mathrm{E}}}=\{0\}$. In $\mathbb{F}_{p^{2 \nu}}[G]$, the Hermitian inner product between $u=\sum_{g \in G} u_{g} Y^{g}$ and $v=\sum_{g \in G} v_{g} Y^{g} \mathbb{F}_{p^{2 \nu}}[G]$ is defined to be $\langle u, v\rangle_{\mathrm{H}}:=\sum_{g \in G} u_{g} v_{g}^{p^{\nu}}$. The Hermitian dual of an abelian code $C$ in $\mathbb{F}_{p^{2 \nu}}[G]$ is defined to be $C^{\perp_{\mathrm{H}}}:=\left\{v \in \mathbb{F}_{p^{2 \nu}}[G] \mid\langle c, v\rangle_{\mathrm{H}}=0\right.$ for all $\left.c \in C\right\}$. An abelian code $C$ in $\mathbb{F}_{p^{2 \nu}}[G]$ is said to be Hermitian complementary dual if $C \cap C^{\perp_{\mathrm{H}}}=\{0\}$.

For positive integers $i$ and $j$ with $\operatorname{gcd}(i, j)=1$, let $\operatorname{ord}_{j}(i)$ denote the multiplicative order of $i$ modulo $j$. Let $P$ denote the Sylow $p$-subgroup of $G$. Then $G \cong A \times P$, where $A$ is a subgroup $G$ such that $|A|=[G: P]$ and $p \nmid|A|$. For each $a \in A$, denote by $\operatorname{ord}(a)$ the additive order of $a$ in $A$. A $p^{\nu}$-cyclotomic class of $A$ containing $a \in A$, denoted by $S_{p^{\nu}}(a)$, is defined to be the set

$$
S_{p^{\nu}}(a):=\left\{p^{\nu i} \cdot a \mid i=0,1, \ldots\right\}=\left\{p^{\nu i} \cdot a \mid 0 \leq i<\operatorname{ord}_{\text {ord }(a)}\left(p^{\nu}\right)\right\},
$$

where $p^{\nu i} \cdot a:=\sum_{j=1}^{p^{\nu i}} a$ in $A$.

First, we consider the decomposition of $\mathcal{R}:=\mathbb{F}_{p^{\nu}}[A]$. In this case, $\mathcal{R}$ is semisimple (see 2]) which can be decomposed using the Discrete Fourier Transform in [20] (see [13] and [12] for more details). For completeness, the decomposition used in this paper is summarized as follows.

An idempotent in $\mathcal{R}$ is a nonzero element $e$ such that $e^{2}=e$. It is called primitive if for every other idempotent $f$, either $e f=e$ or $e f=0$. The primitive idempotents in $\mathcal{R}$ are induced by the $p^{\nu}$-cyclotomic classes of $A$ (see [7, Proposition II.4]). Let $\left\{a_{1}, a_{2}, \ldots, a_{t}\right\}$ be a complete set of representatives of $p^{\nu}$-cyclotomic classes of $A$ and let $e_{i}$ be the primitive idempotent induced by $S_{p^{\nu}}\left(a_{i}\right)$ for all $1 \leq i \leq t$. From [20], $\mathcal{R}$ can be decomposed as

$$
\mathcal{R}=\bigoplus_{i=1}^{t} \mathcal{R} e_{i}
$$

It is well known (see [12] and [13]) that $\mathcal{R} e_{i} \cong \mathbb{F}_{p^{\nu s_{i}}}$, where $s_{i}=\left|S_{p^{\nu}}\left(a_{i}\right)\right|$ provided that $e_{i}$ is induced by $S_{p^{\nu}}\left(a_{i}\right)$, and hence,

$$
\mathbb{F}_{p^{\nu}}[A \times P] \cong \bigoplus_{i=1}^{t}\left(\mathcal{R} e_{i}\right)[P] \cong \prod_{i=1}^{t} \mathbb{F}_{p^{\nu s_{i}}}[P] .
$$

Therefore, every abelian code $C$ in $\mathbb{F}_{p^{\nu}}[A \times P]$ can be viewed as

$$
C \cong \prod_{i=1}^{t} C_{i}
$$

where $C_{i}$ is an abelian code in $\mathbb{F}_{p^{\nu s_{i}}}[P]$ for all $i=1,2, \ldots, t$.

The goal of this paper is to characterize and enumerate complementary dual abelian codes in $\mathbb{F}_{p^{\nu}}[G]$ for all finite abelian groups $G$, primes $p$, and positive integers $\nu$. The characterization of each component in the decomposition (3) is given in Section 3. Combining the results in Sections 2 and 3, the characterization and enumeration of complementary dual abelian codes in $\mathbb{F}_{p^{\nu}}[G]$ are given in Sections 4-5. 


\section{Abelian Codes in some Local Group Algebras}

In this section, we focus on complementary dual abelian codes and direct summand ideals in each component $\mathbb{F}_{p^{\nu}}[P]$ in the decomposition (3).

For a finite commutative ring $R$ with identity, the Jacobson radical of $R$, denoted by $\operatorname{Jac}(R)$, is defined to be the intersection of all maximal ideals of $R$. The ring $R$ is said to be local if it has a unique maximal ideal and it is called a chain ring if its ideals are linearly ordered by inclusion. A finite commutative chain ring $R$ with maximal ideal $M$ is said to have nilpotency index $e$ if $e$ is the smallest positive integer such that $M^{e}=0$. The following facts are well known.

Proposition 3.1. A finite commutative ring $R$ with identity is a local ring if and only if the set of all non-invertible elements in $R$ forms a maximal ideal.

Proposition 3.2. A finite commutative local ring $R$ is a chain ring if and only if its maximal ideal is principal.

A local group ring has been characterized in the following lemma.

Lemma 3.3 ( [18, Theorem]). Let $R$ be a commutative ring with identity and let $G$ be a finite abelian group. Then $R[G]$ is local if and only if $R$ is local, $G$ is a p-group and $p \in \operatorname{Jac}(R)$, where $\operatorname{Jac}(R)$ is the Jacobson radical of $R$.

From Lemma 3.3 , a group algebra $\mathbb{F}_{p^{\nu}}[P]$ is local for all $p$-groups $P$. More properties of a local group algebra $\mathbb{F}_{p^{\nu}}[P]$ can be deduced as follows.

Proposition 3.4. Let $p$ be a prime and let $\nu$ be a positive integer. Let $P$ be a finite abelian p-group. Then the following statements holds.

1. $\mathbb{F}_{p^{\nu}}[P]$ is a local group algebra with maximal ideal $\Delta_{\mathbb{F}_{p^{\nu}}}(P)$.

2. $\mathbb{F}_{p^{\nu}}\left[\mathbb{Z}_{p^{k}}\right]$ is a finite chain ring with nilpotency index $p^{k}$ for all positive integers $k$. .

Proof. Since $\mathbb{F}_{p^{\nu}}$ is a field of characteristic $p, \mathbb{F}_{p^{\nu}}$ is local and $p \in\{0\}=\operatorname{Jac}\left(\mathbb{F}_{p^{\nu}}\right)$. By Lemma 3.3, $\mathbb{F}_{p^{\nu}}[P]$ is local. Using the map $\epsilon$ defined in (11) and the first isomorphism for rings, we have

$$
\mathbb{F}_{p^{\nu}} \cong \mathbb{F}_{p^{\nu}}[P] / \operatorname{ker}(\epsilon) \cong \mathbb{F}_{p^{\nu}}[P] / \Delta_{\mathbb{F}_{p^{\nu}}}(P),
$$

and hence, $\Delta_{\mathbb{F}_{p^{\nu}}}(P)$ is the maximal ideal of $\mathbb{F}_{p^{\nu}}[P]$. This completes the proof of 1 .

It is not difficult to see that the maximal ideal $\Delta_{\mathbb{F}_{p^{\nu}}}\left(\mathbb{Z}_{p^{k}}\right) \cong\langle Y-1\rangle$ is principal. By Proposition $\left[3.2, \mathbb{F}_{p^{\nu}}\left[\mathbb{Z}_{p^{k}}\right]\right.$ is a finite chain ring. Since $(Y-1)^{p^{k}}=0$ and $(Y-$ $1)^{p^{k}-1} \neq 0, \mathbb{F}_{p^{\nu}}\left[\mathbb{Z}_{p^{k}}\right]$ has nilpotency index $p^{k}$. This completes the proof of 2 .

Corollary 3.5. Let $p$ be a prime and let $\nu$ be a positive integer. Then $\mathbb{F}_{p^{\nu}}\left[\mathbb{Z}_{p^{k}}\right]$ contains $p^{k}+1$ ideals.

Proof. The statement follows from Proposition 3.4. Precisely, the ideals in $\mathbb{F}_{p^{\nu}}\left[\mathbb{Z}_{p^{k}}\right]$ are of the form $\left\langle(Y-1)^{i}\right\rangle$ for all $0 \leq i \leq p^{k}$.

The characterizations of the Euclidean and Hermitian complementary dual abelian codes and the direct summands in a local group algebra $\mathbb{F}_{p^{\nu}}[P]$ are given in the following theorems. 
Theorem 3.6. Let $p$ be a prime and let $\nu \geq 1$ be an integer. Let $P$ be a finite abelian p-group. Then $\{0\}$ and $\mathbb{F}_{p^{\nu}}[P]$ are the only Euclidean complementary dual abelian codes in $\mathbb{F}_{p^{\nu}}[P]$.

Proof. Clearly, $\{0\}$ and $\mathbb{F}_{p^{\nu}}[P]$ are Euclidean complementary dual abelian codes in $\mathbb{F}_{p^{\nu}}[P]$. Assume that there exists a Euclidean abelian code $C$ in $\mathbb{F}_{p^{\nu}}[P]$ such that $\{0\} \subsetneq C \subsetneq \mathbb{F}_{p^{\nu}}[P]$. By Proposition $\left[3.4, \mathbb{F}_{p^{\nu}}[P]\right.$ is local with maximal ideal $\Delta_{\mathbb{F}_{p^{\nu}}}(P)$. Then $C \subseteq \Delta_{\mathbb{F}_{p^{\nu}}}(P)$. It follows that $\Delta_{\mathbb{F}_{p^{\nu}}}(P)^{\perp_{\mathrm{E}}} \subseteq C^{\perp_{\mathrm{E}}} \subseteq \Delta_{\mathbb{F}_{p^{\nu}}}(P)$ which implies $\Delta_{\mathbb{F}_{p^{\nu}}}(P)^{\perp_{\mathrm{E}}} \subseteq C \subseteq \Delta_{\mathbb{F}_{p^{\nu}}}(P)$. Hence, $\{0\} \neq \Delta_{\mathbb{F}_{p^{\nu}}}(P)^{\perp_{\mathrm{E}}} \subseteq C \cap C^{\perp_{\mathrm{E}}} \subseteq \Delta_{\mathbb{F}_{p^{\nu}}}(P)$. Consequently, $C$ is not Euclidean complementary dual. Therefore, the ideals $\{0\}$ and $\mathbb{F}_{p^{\nu}}[P]$ are the only Euclidean complementary dual abelian codes in $\mathbb{F}_{p^{\nu}}[P]$.

It is not difficult to see that the proof of Theorem 3.6 is independent of the inner product. Hence, we have the following corollary.

Corollary 3.7. Let $p$ be a prime and let $\nu$ be a positive integer. Let $P$ be a finite abelian p-group. Then $\{0\}$ and $\mathbb{F}_{p^{2 \nu}}[P]$ are the only Hermitian complementary dual abelian codes in $\mathbb{F}_{p^{2 \nu}}[P]$.

Theorem 3.8. Let $p$ be a prime and let $\nu$ be a positive integer. Let $P$ be a finite abelian p-group. Then ideals $\{0\}$ and $\mathbb{F}_{p^{\nu}}[P]$ are the only direct summands in $\mathbb{F}_{p^{\nu}}[P]$.

Proof. Let $\{0\} \subsetneq C \subsetneq \mathbb{F}_{p^{\nu}}[B]$ be an ideal in $\mathbb{F}_{p^{\nu}}[P]$. Suppose that $C$ is a direct summand. Then there exist an ideal $C^{\prime}$ in $\mathbb{F}_{p^{\nu}}[P]$ such that $C \cap C^{\prime}=\{0\}$ and $C+C^{\prime}=\mathbb{F}_{p^{\nu}}[P]$. Since $\Delta_{\mathbb{F}_{p^{\nu}}}(P)$ is the maximal ideal in $\mathbb{F}_{p^{\nu}}[P]$, we have $C^{\prime} \subseteq$ $\Delta_{\mathbb{F}_{p^{\nu}}}(P)$. Hence, $C+C^{\prime} \subseteq \Delta_{\mathbb{F}_{p^{\nu}}}(P) \subsetneq \mathbb{F}_{p^{\nu}}[P]$, a contradiction. Therefore, the ideals $\{0\}$ and $\mathbb{F}_{p^{\nu}}[P]$ are the only direct summands in $\mathbb{F}_{p^{\nu}}[P]$.

The next corollary follows immediately.

Corollary 3.9. Let $p$ be a prime and let $\nu$ be a positive integer. Let $P$ be a finite abelian p-group. Then the following statements hold.

1. The number of Euclidean complementary dual abelian codes in $\mathbb{F}_{p^{\nu}}[P]$ is 2 .

2. The number of direct summand ideals in $\mathbb{F}_{p^{\nu}}[P]$ is 2 .

3. The number of Hermitian complementary dual abelian codes in $\mathbb{F}_{p^{2 \nu}}[P]$ is 2 .

4. The number of direct summand ideals in $\mathbb{F}_{p^{2 \nu}}[P]$ is 2 .

\section{Euclidean Complementary Dual Abelian Codes}

In this section, we focus on characterization and enumeration of Euclidean complementary dual abelian codes in $\mathbb{F}_{p^{\nu}}[G]$, where $p$ is a prime, $\nu$ is a positive integer, and $G$ is an arbitrary finite abelian group. 


\subsection{Characterization}

For a finite abelian group $G$, write $G=A \times P$, where $p \nmid|A|$ and $P$ is the Sylow $p$-subgroup of $G$. Based on the results in Section 3, the characterization and enumeration of complementary dual abelian codes in $\mathbb{F}_{p^{\nu}}[G]$ are given and they are shown to be independent of $P$.

The decomposition of $\mathbb{F}_{p^{\nu}}[A]$ in [12] plays a vital role in the study of Euclidean complementary dual abelian codes in $\mathbb{F}_{p^{\nu}}[G]$ and it is recalled as follows. A $p^{\nu}$ cyclotomic class $S_{p^{\nu}}(a)$ is said to be of type $I$ if $S_{p^{\nu}}(a)=S_{p^{\nu}}(-a)$, or type II if $S_{p^{\nu}}(-a) \neq S_{p^{\nu}}(a)$. Without loss of generality, the representatives $a_{1}, a_{2}, \ldots, a_{t}$ of $p^{\nu}$-cyclotomic classes of $A$ can be chosen such that $\left\{a_{j} \mid j=1,2, \ldots, r_{I}\right\}$ and $\left\{a_{r_{I}+l}, a_{r_{I}+r_{I}+l}=-a_{r_{I}+l} \mid l=1,2, \ldots, r_{I I}\right\}$ are sets of representatives of $p^{\nu}$-cyclotomic classes of $A$ of types $I$ and $I I$, respectively, where $t=r_{I}+2 r_{I I}$. Assume the notations used in Section 2, we have $s_{i}=\left|S_{p^{\nu}}\left(a_{i}\right)\right|$ for all $1 \leq i \leq t$ and $s_{r_{I}+l}=s_{r_{I}+r_{I}+l}$ for all $1 \leq l \leq r_{I I}$

Rearranging the terms in the decomposition of $\mathcal{R}$ in (3) based on these 2 types of cyclotomic classes (see [12]), we have

$$
\mathbb{F}_{p^{\nu}}[A \times P] \cong\left(\prod_{j=1}^{r_{I}} \mathbb{K}_{j}[P]\right) \times\left(\prod_{l=1}^{r_{I}}\left(\mathbb{L}_{l}[P] \times \mathbb{L}_{l}[P]\right)\right),
$$

where $\mathbb{K}_{j} \cong \mathbb{F}_{p^{\nu s_{j}}}$ for all $j=1,2, \ldots, r_{I}$ and $\mathbb{L}_{l} \cong \mathbb{F}_{p^{\nu s r_{I}+l}}$ for all $l=1,2, \ldots, r_{I}$.

From (5), it follows that an abelian code $C$ in $\mathbb{F}_{p^{\nu}}[A \times P]$ can be viewed as

$$
C \cong\left(\prod_{j=1}^{r_{I}} C_{j}\right) \times\left(\prod_{l=1}^{r_{I}}\left(D_{l} \times D_{l}^{\prime}\right)\right)
$$

where $C_{j}, D_{s}$ and $D_{s}^{\prime}$ are abelian codes in $\mathbb{K}_{j}[P], \mathbb{L}_{l}[P]$ and $\mathbb{L}_{l}[P]$, respectively, for all $j=1,2, \ldots, r_{I}$ and $l=1,2, \ldots, r_{I I}$.

From [12, Section II.D], the Euclidean dual of $C$ in (6) is of the form

$$
C^{\perp_{\mathrm{E}}} \cong\left(\prod_{j=1}^{r_{I}} C_{j}^{\perp_{\mathrm{H}}}\right) \times\left(\prod_{l=1}^{r_{I}}\left(\left(D_{l}^{\prime}\right)^{\perp_{\mathrm{E}}} \times D_{l}^{\perp_{\mathrm{E}}}\right)\right),
$$

where $\perp_{\mathrm{H}}:=\perp_{\mathrm{E}}$ if $\mathbb{K}_{j} \cong \mathbb{F}_{p^{k}}$.

The characterization of a Euclidean complementary dual abelian code in $\mathbb{F}_{p^{\nu}}[G]$ is given in the following proposition.

Proposition 4.1. Let $p$ be a prime and let $\nu$ be a positive integer. Let $A$ be finite abelian group such that $p \nmid|A|$ and let $P$ be a finite abelian p-group. Then an abelian code $C$ in $\mathbb{F}_{p^{\nu}}[A \times P]$ decomposed as in (6) is Euclidean complementary dual if and only if the following statements hold.

1. $C_{j}$ is Hermitian complementary dual for all $1 \leq j \leq r_{I}$.

2. $D_{l} \cap\left(D_{l}^{\prime}\right)^{\perp_{\mathrm{E}}}=\{0\}$ and $D_{l}^{\prime} \cap D_{l}^{\perp_{\mathrm{E}}}=\{0\}$ for all $1 \leq l \leq r_{I I}$.

Proof. The result can be deduced directly from (6) and (77). 
Corollary 4.2. Let $p$ be a prime and let $\nu$ be a positive integer. Let $A$ be finite abelian group such that $p \nmid|A|$ and let $P$ be a finite abelian p-group. Then an abelian code $C$ in $\mathbb{F}_{p^{\nu}}[A \times P]$ decomposed as in (6) is Euclidean complementary dual if and only if the following statements hold.

1. $C_{j} \in\left\{\{0\}, \mathbb{K}_{j}[P]\right\}$ for all $1 \leq j \leq r_{I}$.

2. $\left(D_{l}, D_{l}^{\prime}\right) \in\left\{\left(\{0\}, \mathbb{L}_{j}[P]\right),\left(\mathbb{L}_{j}[P],\{0\}\right)\right\}$ for all $1 \leq l \leq r_{I I}$.

Proof. From Proposition 4.1, $C_{j}$ is Hermitian complementary dual for all $1 \leq j \leq r_{I}$ and $D_{l}$ is a direct summand for all $1 \leq l \leq r_{I I}$. By Corollary [3.7, $C_{j} \in\left\{\{0\}, \mathbb{K}_{j}[P]\right\}$ for all $1 \leq j \leq r_{I}$. By Theorem 3.8, $D_{l} \in\left\{\{0\}, \mathbb{L}_{l}[P]\right\}$ for all $1 \leq l \leq r_{I I}$, and hence, the result follows.

From Corollary 4.2, it is not difficult to see that the number of Euclidean complementary dual abelian codes in $\mathbb{F}_{p^{\nu}}[A \times P]$ is independent of $P$ and it is determined in the following corollary.

Corollary 4.3. Let $p$ be a prime and let $\nu$ be a positive integer. Let $A$ be finite abelian group such that $p \nmid|A|$ and let $P$ be a finite abelian p-group. If $\mathbb{F}_{p^{\nu}}[A \times P]$ decomposed as in (5), then the number of Euclidean complementary dual abelian codes in $\mathbb{F}_{p^{\nu}}[A \times P]$ is

$$
2^{r_{I}+r_{I I}}
$$

Proof. From the characterization in Corollary [4.2, the number of choices of $C_{j}$ is $2^{r_{I}}$ and the number of choices of $\left(D_{l}, D_{l}^{\prime}\right)$ is $2^{r_{I I}}$. Hence, the number of Euclidean complementary dual abelian codes in $\mathbb{F}_{p^{\nu}}[A \times P]$ is $2^{r_{I}+r_{I I}}$ as desired.

\subsection{Enumerations}

As discussed in Corollary 4.3, the number of Euclidean complementary dual abelian codes in $\mathbb{F}_{p^{\nu}}[A \times P]$ is independent of $P$. Here, it is sufficient to determined the number $r_{I}+r_{I I}$ from the group algebra $\mathbb{F}_{p^{\nu}}[A]$.

Let $\chi$ be a function defined by

$$
\chi\left(d, p^{\nu}\right)= \begin{cases}1 & \text { if there exists a positive integer } i \text { such that } d \mid\left(p^{\nu i}+1\right), \\ 0 & \text { otherwise. }\end{cases}
$$

The following lemma is a straightforward generalization of the case where $p=2$ (see [12, Lemma 4.5]).

Lemma 4.4. Let $p$ be a prime and let $\nu$ be a positive integer. Let $A$ be a finite abelian group such that $p \nmid|A|$ and let $a \in A$. Then $S_{p^{\nu}}(a)$ is of type $I$ if and only if $\chi\left(\operatorname{ord}(a), p^{\nu}\right)=1$.

The value $r_{I}+r_{I I}$ of the group algebra $\mathbb{F}_{p^{\nu}}[A]$ is determined in the following proposition.

Proposition 4.5. Let $p$ be a prime and let $\nu$ be a positive integer. Let $A$ be a finite abelian group of exponent $N$. If $p \nmid N$, then

$$
r_{I}+r_{I I}=\sum_{d \mid N} \chi\left(d, p^{\nu}\right) \frac{\mathcal{N}_{A}(d)}{\operatorname{ord}_{d}\left(p^{\nu}\right)}+\frac{1}{2} \sum_{d \mid N}\left(1-\chi\left(d, p^{\nu}\right)\right) \frac{\mathcal{N}_{A}(d)}{\operatorname{ord}_{d}\left(p^{\nu}\right)},
$$

where $\mathcal{N}_{A}(d)$ denotes the number of elements of order $d$ in $A$ determined in [1]. 
Proof. Using the arguments similar to those in [13, Remark 2.5], for each $d \mid N$, the elements of order $d$ in $A$ are partitioned into $p^{\nu}$-cyclotomic classes of the same size $\frac{\mathcal{N}_{A}(d)}{\operatorname{ord}_{d}\left(p^{\nu}\right)}$. By Lemma 4.4, it follows that

$$
r_{I}=\sum_{d \mid N} \chi\left(d, p^{\nu}\right) \frac{\mathcal{N}_{A}(d)}{\operatorname{ord}_{d}\left(p^{\nu}\right)} \text { and } r_{I I}=\frac{1}{2} \sum_{d \mid N}\left(1-\chi\left(d, p^{\nu}\right)\right) \frac{\mathcal{N}_{A}(d)}{\operatorname{ord}_{d}\left(p^{\nu}\right)} .
$$

Hence, the result follows.

In the case where $A$ is a cyclic group of order $n$ with $p \nmid n$, it follows that the exponent of $A$ is $n$ and $\mathcal{N}_{A}(d)=\Phi(d)$ for all divisors $d$ of $n$, where $\Phi(d)$ denotes the Euler totient phi function. Hence, the following corollary can be deduced.

Corollary 4.6. Let $p$ be a prime and let $\nu$ be a positive integer. Let $n$ be a positive integer such that $p \nmid n$. Then the number of Euclidean complementary dual cyclic codes of length $n p^{k}$ over $\mathbb{F}_{p^{\nu}}$ equals the number of Euclidean complementary dual cyclic codes of length $n$ over $\mathbb{F}_{p^{\nu}}$ for all integers $k \geq 0$ which is

$$
2^{\sum_{d \mid n} \chi\left(d, p^{\nu}\right) \frac{\Phi(d)}{\operatorname{ord}_{d}\left(p^{\nu}\right)}+\frac{1}{2} \sum_{d \mid n}\left(1-\chi\left(d, p^{\nu}\right)\right) \frac{\Phi(d)}{\operatorname{ord}_{d}\left(p^{\nu}\right)}} .
$$

From Proposition 4.5, for an arbitrary finite abelian group $A$ with $p \nmid|A|$, we have $r_{I}+r_{I I} \geq 1$. Hence, there exist at least two Euclidean complementary dual abelian codes in $\mathbb{F}_{p^{\nu}}[A]$.

In Table 2, the number $r_{I}+r_{I I}$ for group algebras $\mathbb{F}_{2}[A]$ is given for abelian groups $A$ of odd order less than 50 .

In the following subsections, a simplified formula for (8) is given for some families of finite abelian $q$-groups, where $q$ is a prime such that $p \neq q$. Let $A \cong\left(\mathbb{Z}_{q^{k}}\right)^{s}$, where $k$ and $s$ are positive integers, and $q$ is prime such that $\operatorname{gcd}(p, q)=1$. For each $0 \leq i \leq k$, define

$$
A_{q^{i}}:=\left\{a \in A \mid \operatorname{ord}(a)=q^{i}\right\} .
$$

Clearly, $A_{1}, A_{q}, \ldots, A_{q^{k}}$ are pair-wise disjoint and $A=A_{1} \cup A_{q} \cup \cdots \cup A_{q^{k}}$. For each $1 \leq i \leq k$, it is not difficult to see that $A_{q^{i}}=\left(q^{k-i} \mathbb{Z}_{q^{k}}\right)^{s} \backslash\left(q^{k-(i-1)} \mathbb{Z}_{q^{k}}\right)^{s}$. Consequently, we have $\left|A_{1}\right|=1$ and

$$
\left|A_{q^{i}}\right|=q^{i s}-q^{(i-1) s}
$$

for all $i=1,2, \ldots, k$. Note that $\left|S_{p^{\nu}}(a)\right|=\operatorname{ord}_{\text {ord }(a)}\left(p^{\nu}\right)=\operatorname{ord}_{q^{i}}\left(p^{\nu}\right)$ for all $a \in A_{q^{i}}$.

\subsection{1 $A=\left(\mathbb{Z}_{2^{k}}\right)^{s}$ and $p$ is an odd prime}

Here, we consider the case where $p$ is odd and $q=2$, i.e., $A \cong\left(\mathbb{Z}_{2^{k}}\right)^{s}$. Clearly, $A_{1}=\{0\}$ and $S_{p^{\nu}}(0)$ is of type $I$. For an element $a \in A_{2}$, we have $\operatorname{ord}(a)=2$ which implies that $a=-a$. Hence, $S_{p^{\nu}}(a)=S_{p^{\nu}}(-a)$ is of type $I$ and its cardinality is 1 . In general, we have the following characterizations of the $p^{\nu}$-cyclotomic classes of type $I$ in $A$.

Lemma 4.7. For each $i \in\{0,1,2, \ldots, k\}$, let $a \in A_{2^{i}}$. Then one of the following statements holds.

1. If $i \in\{0,1\}$, then $S_{p^{\nu}}(a)$ is of type $I$ and size 1 . 


\begin{tabular}{|r|r|r|}
\hline Order of $A$ & \multicolumn{1}{|r|}{$A$} & $r_{I}+r_{I I}$ \\
\hline 3 & $\mathbb{Z}_{3}$ & 2 \\
5 & $\mathbb{Z}_{5}$ & 2 \\
7 & $\mathbb{Z}_{7}$ & 2 \\
9 & $\mathbb{Z}_{3} \times \mathbb{Z}_{3}$ & 5 \\
& $\mathbb{Z}_{3^{2}}$ & 3 \\
11 & $\mathbb{Z}_{11}$ & 2 \\
13 & $\mathbb{Z}_{13}$ & 2 \\
15 & $\mathbb{Z}_{5} \times \mathbb{Z}_{3}$ & 4 \\
17 & $\mathbb{Z}_{17}$ & 3 \\
19 & $\mathbb{Z}_{19}$ & 2 \\
21 & $\mathbb{Z}_{7} \times \mathbb{Z}_{3}$ & 4 \\
23 & $\mathbb{Z}_{23}$ & 2 \\
25 & $\mathbb{Z}_{5} \times \mathbb{Z}_{5}$ & 7 \\
& $\mathbb{Z}_{5^{2}}$ & 3 \\
27 & $\mathbb{Z}_{3} \times \mathbb{Z}_{3} \times \mathbb{Z}_{3}$ & 14 \\
& $\mathbb{Z}_{32} \times \mathbb{Z}_{3}$ & 8 \\
& $\mathbb{Z}_{33}$ & 4 \\
29 & $\mathbb{Z}_{29}$ & 2 \\
31 & $\mathbb{Z}_{31}$ & 4 \\
33 & $\mathbb{Z}_{11} \times \mathbb{Z}_{3}$ & 5 \\
35 & $\mathbb{Z}_{7} \times \mathbb{Z}_{5}$ & 4 \\
37 & $\mathbb{Z}_{37}$ & 2 \\
39 & $\mathbb{Z}_{13} \times \mathbb{Z}_{3}$ & 4 \\
41 & $\mathbb{Z}_{41}$ & 3 \\
43 & $\mathbb{Z}_{43}$ & 4 \\
45 & $\mathbb{Z}_{5} \times \mathbb{Z}_{3} \times \mathbb{Z}_{3}$ & 10 \\
47 & $\mathbb{Z}_{5} \times \mathbb{Z}_{32}$ & 6 \\
49 & $\mathbb{Z}_{47}$ & 3 \\
& $\mathbb{Z}_{7} \times \mathbb{Z}_{7}$ & 9 \\
& $\mathbb{Z}_{7^{2}}$ & 3 \\
\hline
\end{tabular}

Table 1: The number $r_{I}+r_{I I}$ for group algebra $\mathbb{F}_{2}[A]$

2. If $i \geq 2$, then $S_{p^{\nu}}(a)$ is of type I if and only if $\operatorname{ord}_{2^{i}}\left(p^{\nu}\right)=2$.

Proof. The first statement follows from the discussion above. To prove the second statement, assume that $i \geq 2$. Assume that $S_{p^{\nu}}(a)$ is of type $I$. Then $-a \in S_{p^{\nu}}(a)$. Then $p^{\nu j} \cdot a=-a$ for some integer $j \geq 1$. Since $i \geq 2$, we have $4 \mid\left(p^{\nu j}+1\right)$. If $j$ is even, then $p^{\nu j} \equiv 1 \bmod 4$ which implies that $\left(p^{\nu j}+1\right) \equiv 2 \bmod 4$, a contradiction. It follows that $j$ is odd. Since $p^{\nu j}+1=\left(p^{\nu j}+1\right)\left(\sum_{i=0}^{j-1}(-1)^{i} p^{\nu(j-1-i)}\right)$ and $\sum_{i=0}^{j-1}(-1)^{i} p^{\nu(j-1-i)}$ is odd, we have $2^{i} \mid\left(p^{\nu}+1\right)$. Hence, $p^{\nu} \equiv-1 \bmod 2^{i}$ which implies that $\operatorname{ord}_{2^{i}}\left(p^{\nu}\right)=2$.

Conversely, assume that $\operatorname{ord}_{2^{i}}\left(p^{\nu}\right)=2$. Then $p^{2 \nu} \cdot a=a$ and $p^{\nu} \cdot a \neq a$ which implies that $p^{\nu} \cdot a=-a$. Hence, $S_{p^{\nu}}(a)$ is a $p^{\nu}$-cyclotomic class of type $I$.

Proposition 4.8. Let $0 \leq r^{\prime} \leq k$ be the largest integer such that $2^{r^{\prime}} \mid\left(p^{\nu}+1\right)$ and let $a \in A$. Then $S_{p^{\nu}}(a)$ is of type $I$ if and only if $a \in A_{2^{i}}$ for some $0 \leq i \leq r^{\prime}$. 
Equivalently, $a \in A$ is of type II if and only if $a \in A_{2^{i}}$ for some $i>r^{\prime}$.

Proof. Assume that $i>r^{\prime}$. Then $2^{i} \nmid\left(p^{\nu}+1\right)$ which implies that $\operatorname{ord}_{2^{i}}\left(p^{\nu}\right)>2$. By Lemma 4.7, $S_{p^{\nu}}(a)$ is not of type $I$.

Conversely, assume that $a \in A_{2^{i}}$ for some $0 \leq i \leq r^{\prime}$. From Lemma 4.7, if $i \in\{0,1\}$, then $S_{p^{\nu}}(a)$ is of type $I$. Assume that $2 \leq i \leq r^{\prime}$. Then $2^{i} \geq 4$ and $2^{i} \mid\left(p^{\nu}+1\right)$ which implies that $\operatorname{ord}_{2^{i}}\left(p^{\nu}\right)=2$. By Lemma 4.7 it follows that $S_{p^{\nu}}(a)$ is of type $I$.

Using Proposition 4.8, $A_{2^{i}}$ contains $p^{\nu}$-cyclotomic classes of type $I$ for all $i=$ $0,1, \ldots, r^{\prime}$ and the rest of the sets $A_{2^{j}}$ contain $p^{\nu}$-cyclotomic classes of type $I I$, for $j=r^{\prime}+1, \ldots, k$. Since $\operatorname{ord}_{2^{i}}\left(p^{\nu}\right)=1$ for all $i \in\{0,1\}$ and $\operatorname{ord}_{2^{i}}\left(p^{\nu}\right)=2$ for all $2 \leq i \leq r^{\prime}$, we have

$$
r_{I}=\sum_{i=0}^{r^{\prime}} \frac{\left|A_{2^{i}}\right|}{\mathrm{ord}_{2^{i}}\left(p^{\nu}\right)}=\left|A_{1}\right|+\left|A_{2}\right|+\sum_{i=2}^{r^{\prime}} \frac{\left|A_{2^{i}}\right|}{2}=2^{s}+\frac{2^{r^{\prime} s}-2^{s}}{2}
$$

and

$$
r_{I I}=\sum_{i=r^{\prime}+1}^{k} \frac{\left|H_{2^{i}}\right|}{20 r d_{2^{i}}\left(p^{\nu}\right)}=\sum_{i=r^{\prime}+1}^{k} \frac{2^{i s}-2^{(i-1) s}}{2 \mathrm{o} r d_{2^{i}}\left(p^{\nu}\right)} .
$$

Hence, the number of Euclidean complementary dual abelian codes in $\mathbb{F}_{p^{\nu}}\left[\left(\mathbb{Z}_{2^{k}}\right)^{s}\right]$ follows immediately from Corollary 4.3 .

\subsection{2 $A=\left(\mathbb{Z}_{q^{k}}\right)^{s}$, where $q$ is an odd prime and $\operatorname{gcd}(q, p)=1$}

In this part, we focus on the case where $A=\left(\mathbb{Z}_{q^{k}}\right)^{s}$, where $q$ is an odd prime and $\operatorname{gcd}(q, p)=1$. Note that $S_{p^{\nu}}(0)=\{0\}=A_{1}$ is of type $I$.

Next, we characterize the type of $p^{\nu}$-cyclotomic classes of elements in $A_{q}$ in terms of $\operatorname{ord}_{q}\left(p^{\nu}\right)$.

Lemma 4.9 ( [15, Proposition 4]). Let $q$ be an odd prime and let $i$ be a positive integer. If $p$ is a prime such that $q \neq p$, then $\operatorname{ord}_{q^{i}}(p)=\operatorname{ord}_{q}(p) q^{j}$ for some $j \geq 0$.

Proposition 4.10. Let $a \in A_{q}$. Then $S_{p^{\nu}}(a)$ is of type I if and only if $\operatorname{ord}_{q}\left(p^{\nu}\right)$ is even. Equivalently, $S_{p^{\nu}}(a)$ is of type II if and only if $\operatorname{ord}_{q}\left(p^{\nu}\right)$ is odd.

Proof. The first implication follows from [11, Remark 3.1].

Conversely, assume that $\operatorname{ord}_{q}\left(p^{\nu}\right)=2 j$ is even, where $j$ is a positive integer. Then $a=p^{\nu \operatorname{ord}_{q}\left(p^{\nu}\right)} \cdot a=p^{2 \nu j} \cdot a$, i.e., $\left(p^{\nu j}-1\right)\left(p^{\nu j}+1\right) \cdot a=0$. Since $\operatorname{ord}_{q}\left(p^{\nu}\right)=2 j$, we have $q \nmid\left(p^{\nu j}-1\right)$, and hence, $\left(p^{\nu j}+1\right) \cdot a=0$. This implies that $-a=p^{\nu j} \cdot a \in S_{p^{\nu}}(a)$. Therefore, $S_{p^{\nu}}(a)$ is of type $I$ as desired.

Next, we show that all $p^{\nu}$-cyclotomic classes of elements in $A \backslash\{0\}$ are of the same type.

Proposition 4.11. Let $a \in A_{q}$ and $b \in A_{q^{i}}$, for any given $1 \leq i \leq k$. Then, $S_{p^{\nu}}(a)$ is of type I if and only if $S_{p^{\nu}}(b)$ is of type I. Equivalently, $S_{p^{\nu}}(a)$ is of type II if and only if $S_{p^{\nu}}(b)$ is of type II. 
Proof. Assume that $S_{p^{\nu}}(a)$ is of type $I$. By Proposition 4.10, it follows that $\operatorname{ord}_{q}\left(p^{\nu}\right)$ is even. By Lemma 4.9, $\operatorname{ord}_{q^{i}}\left(p^{\nu}\right)=\operatorname{ord}_{q}\left(p^{\nu}\right) q^{j}$ for some positive integer $j$. Then $q^{i} \mid\left(p^{\nu \operatorname{ord}_{q}\left(p^{\nu}\right) q^{j}}-1\right)$ and $q^{i} \nmid\left(p^{\nu \frac{\operatorname{ord} q\left(p^{\nu}\right)}{2}} q^{j}-1\right)$. If $q \mid\left(p^{\nu \frac{\operatorname{ord} q\left(p^{\nu}\right)}{2}} q^{j}-1\right)$, then $\operatorname{ord}_{q}\left(p^{\nu}\right) \mid \frac{\operatorname{ord}_{q}\left(p^{\nu}\right)}{2} q^{j}$ which is impossible since $q$ is odd. Hence, we have $q^{i} \mid\left(p^{\nu \frac{\operatorname{ord} q\left(p^{\nu}\right)}{2} q^{j}}+1\right)$. Consequently, $-b=p^{\nu \frac{\operatorname{ord} q\left(p^{\nu}\right)}{2} q^{j}} \cdot b$ which means that $S_{p^{\nu}}(b)$ is of type $I$.

Conversely, assume that $S_{p^{\nu}}(b)$ is of type $I$. By [11, Remark 3.1], $\operatorname{ord}_{q^{i}}\left(p^{\nu}\right)$ is even. By Lemma 4.9, it follows that $\operatorname{ord}_{q^{i}}\left(p^{\nu}\right)=\operatorname{ord}_{q}\left(p^{\nu}\right) q^{j}$ for some positive integer $j$. Hence, $\operatorname{ord}_{q}\left(p^{\nu}\right)$ is even. By Proposition 4.10, we have that $S_{p^{\nu}}(a)$ is of type $I$.

Combining Propositions 4.10 and 4.11, the next corollary follows immediately.

Corollary 4.12. Let a be a non-zero element in $A \cong\left(\mathbb{Z}_{q^{k}}\right)^{s}$. Then $S_{p^{\nu}}(a)$ is of type I if and only if $\operatorname{ord}_{q}\left(p^{\nu}\right)$ is even. Equivalently, $S_{p^{\nu}}(a)$ is of type II if and only if $\operatorname{ord}_{q}\left(p^{\nu}\right)$ is odd.

If $\operatorname{ord}_{q}\left(p^{\nu}\right)$ is even, then $S_{p^{\nu}}(a)$ is of type $I$ for all $a \in A \backslash\{0\}$. Hence, by Corollary 4.12 , it can be deduced that

$$
r_{I}=A_{1}+\sum_{i=1}^{k} \frac{\left|A_{q^{i}}\right|}{\operatorname{ord}_{q^{i}}\left(p^{\nu}\right)}=1+\sum_{i=1}^{k} \frac{q^{i s}-q^{(i-1) s}}{\operatorname{ord}_{q^{i}}\left(p^{\nu}\right)} \text { and } r_{I I}=0 .
$$

On the other hand, if $\operatorname{ord}_{q}\left(p^{\nu}\right)$ is odd, then $S_{q}(a)$ is of type $I I$ for all $a \in A \backslash\{0\}$. Hence, by Corollary 4.12, we have

$$
r_{I}=\left|A_{1}\right|=1 \text { and } r_{I I}=\sum_{i=1}^{k} \frac{\left|A_{q^{i}}\right|}{\operatorname{2ord}_{q^{i}}\left(p^{\nu}\right)}=\sum_{i=1}^{k} \frac{q^{i s}-q^{(i-1) s}}{\operatorname{2ord}_{q^{i}}\left(p^{\nu}\right)} .
$$

In both cases, the number of Euclidean complementary dual abelian codes in $\mathbb{F}_{p^{\nu}}\left[\left(\mathbb{Z}_{q^{k}}\right)^{s}\right]$ follows immediately from Corollary 4.3 .

\section{Hermitian Complementary Dual Abelian Codes}

Note that the Hermitian inner product is defined over a finite field of square order. To study Hermitian complementary abelian codes, it suffices to focus on a group algebra $\mathbb{F}_{p^{2 \nu}}[G]$, where $p$ is a prime, $\nu$ is a positive integer, and $G$ is a finite abelian group.

\subsection{Characterization}

For a finite abelian group $G$, write $G=A \times P$, where $p \nmid|A|$ and $P$ is a $p$-group. In this section, the characterization and enumeration of Hermitian complementary dual abelian codes in $\mathbb{F}_{p^{2 \nu}}[G]$ are given and they are shown to be independent of $P$.

The decomposition of $\mathbb{F}_{p^{2 \nu}}[A]$ in $[13$ is key to study Hermitian complementary dual abelian codes in $\mathbb{F}_{p^{2 \nu}}[G]$ and it is recalled as follows. For each $a \in A$, the $p^{2 \nu_{-}}$ cyclotomic class $S_{p^{2 \nu}}(a)$ is said to be of type $I^{\prime}$ if $S_{p^{2 \nu}}(a)=S_{p^{\nu}}\left(-p^{\nu} a\right)$ or type $I I^{\prime}$ if $S_{p^{2 \nu}}(a) \neq S_{p^{2 \nu}}\left(-p^{\nu} a\right)$. Without loss of generality, the representatives $a_{1}, a_{2}, \ldots, a_{t}$ of $p^{2 \nu}$-cyclotomic classes of $A$ can be chosen such that $\left\{a_{j} \mid j=1,2, \ldots, r_{I^{\prime}}\right\}$ and 
$\left\{a_{r_{I^{\prime}}+l}, a_{r_{I}+r_{I^{\prime}}+l}=-p^{\nu} a_{r_{I^{\prime}}+l} \mid l=1,2, \ldots, r_{I^{\prime}}\right\}$ are sets of representatives of $p^{2 \nu_{-}}$ cyclotomic classes of $A$ of types $I^{\prime}$ and $I^{\prime}$, respectively, where $t=r_{I^{\prime}}+2 r_{I^{\prime}}$. Assume the notations used in Section 2, we have $s_{i}=\left|S_{p^{2 \nu}}\left(a_{i}\right)\right|$ for all $1 \leq i \leq t$ and $s_{r_{I^{\prime}}+l}=s_{r_{I^{\prime}}+r_{I^{\prime}}+l}$ for all $1 \leq l \leq r_{I^{\prime}}$.

Rearranging the terms in the decomposition of $\mathcal{R}$ in (3) based on the 2 types of cyclotomic classes mentioned above (see [13]), we have

$$
\mathbb{F}_{p^{2 \nu}}[A \times P] \cong\left(\prod_{j=1}^{r_{I^{\prime}}} \mathbb{K}_{j}[P]\right) \times\left(\prod_{l=1}^{r_{\Pi^{\prime}}}\left(\mathbb{L}_{l}[P] \times \mathbb{L}_{l}[P]\right)\right),
$$

where $\mathbb{K}_{j} \cong \mathbb{F}_{p^{2 \nu s_{j}}}$ for all $j=1,2, \ldots, r_{I^{\prime}}$ and $\mathbb{L}_{l} \cong \mathbb{F}_{p^{2 \nu s_{I_{I^{\prime}}+l}}}$ for all $l=1,2, \ldots, r_{I^{\prime}}$.

From (9), it follows that an abelian code $C$ in $\mathbb{F}_{p^{2 \nu}}[A \times P]$ can be viewed as

$$
C \cong\left(\prod_{j=1}^{r_{I^{\prime}}} C_{j}\right) \times\left(\prod_{l=1}^{r_{\Pi^{\prime}}}\left(D_{l} \times D_{l}^{\prime}\right)\right)
$$

where $C_{j}, D_{s}$ and $D_{s}^{\prime}$ are abelian codes in $\mathbb{K}_{j}[P], \mathbb{L}_{l}[P]$ and $\mathbb{L}_{l}[P]$, respectively, for all $j=1,2, \ldots, r_{I^{\prime}}$ and $l=1,2, \ldots, r_{I^{\prime}}$.

From [13, Section II.D], the Hermitian dual of $C$ in (10) is of the form

$$
C^{\perp_{\mathrm{H}}} \cong\left(\prod_{j=1}^{r_{I^{\prime}}} C_{j}^{\perp_{\mathrm{H}}}\right) \times\left(\prod_{l=1}^{r_{I^{\prime}}}\left(\left(D_{l}^{\prime}\right)^{\perp_{\mathrm{E}}} \times D_{l}^{\perp_{\mathrm{E}}}\right)\right) .
$$

The characterization of Hermitian complementary dual abelian codes in $\mathbb{F}_{p^{2 \nu}}[G]$ is given in the following proposition.

Proposition 5.1. Let $p$ be a prime and let $\nu$ be a positive integer. Let $A$ be a finite abelian group such that $p \nmid|A|$ and let $P$ be a finite abelian p-group. Then an abelian code $C$ in $\mathbb{F}_{p^{2 \nu}}[A \times P]$ decomposed as in (10) is Hermitian complementary dual if and only if the following statements hold.

1. $C_{j}$ is Hermitian complementary dual for all $1 \leq j \leq r_{I^{\prime}}$.

2. $D_{l} \cap\left(D_{l}^{\prime}\right)^{\perp_{\mathrm{E}}}=\{0\}$ and $D_{l}^{\prime} \cap D_{l}^{\perp_{\mathrm{E}}}=\{0\}$ for all $1 \leq l \leq r_{I^{\prime}}$.

Proof. The result can be deduced directly from (10) and (11).

Corollary 5.2. Let $p$ be a prime and let $\nu$ be a positive integer. Let $A$ be a finite abelian group such that $p \nmid|A|$ and let $P$ be a finite abelian p-group. Then an abelian code $C$ in $\mathbb{F}_{p^{2 \nu}}[A \times P]$ decomposed as in (10) is Hermitian complementary dual if and only if the following statements hold.

1. $C_{j} \in\left\{\{0\}, \mathbb{K}_{j}[P]\right\}$ for all $1 \leq j \leq r_{I^{\prime}}$.

2. $\left(D_{l}, D_{l}^{\prime}\right) \in\left\{\left(\{0\}, \mathbb{L}_{j}[P]\right),\left(\mathbb{L}_{j}[P],\{0\}\right)\right\}$ for all $1 \leq l \leq r_{\mathbb{I}^{\prime}}$.

Proof. By Proposition 5.1, $C_{j}$ is Hermitian complementary dual for all $1 \leq j \leq$ $r_{I^{\prime}}$ and $D_{l}$ is a direct summand for all $1 \leq l \leq r_{I^{\prime}}$. By Corollary 3.7, we have $C_{j} \in\left\{\{0\}, \mathbb{K}_{j}[P]\right\}$ for all $1 \leq j \leq r_{I^{\prime}}$. By Theorem $\left[3.8, D_{l} \in\left\{\{0\}, \mathbb{L}_{j}[P]\right\}\right.$ for all $1 \leq l \leq r_{I^{\prime}}$. Hence, the result follows. 
The number of Hermitian complementary dual abelian codes in $\mathbb{F}_{p^{2 \nu}}[A \times P]$ is independent of $P$ and determined in the following corollary.

Corollary 5.3. Let $p$ be a prime and let $\nu$ be a positive integer. Let $A$ be a finite abelian group such that $p \nmid|A|$ and let $P$ be a finite abelian p-group. If $\mathbb{F}_{p^{2 \nu}}[A \times P]$ decomposed as in (9), then the number of Hermitian complementary dual abelian codes in $\mathbb{F}_{p^{2 \nu}}[A \times P]$ is

$$
2^{r_{I^{\prime}}+r_{I^{\prime}}}
$$

Proof. From the characterization in Corollary 5.2, the number of choices of $C_{j}$ is $2^{r_{I^{\prime}}}$ and the number of choices of $\left(D_{l}, D_{l}^{\prime}\right)$ is $2^{r} I^{\prime}$. Hence, the number of Hermitian complementary dual abelian codes in $\mathbb{F}_{p^{2 \nu}}[A \times P]$ is $2^{r_{I}+r_{I I}}$ as desired.

\subsection{Enumerations}

As discussed in Corollary 5.3 , the number of Hermitian complementary dual abelian codes in $\mathbb{F}_{p^{2 \nu}}[A \times P]$ is independent of $P$. It is therefore sufficient to determined the number $r_{I^{\prime}}+r_{I^{\prime}}$ from the semi-simple group algebra $\mathbb{F}_{p^{2 \nu}}[A]$.

Let $\lambda$ be a function defined by

$$
\lambda\left(d, p^{\nu}\right)= \begin{cases}1 & \text { if there exists an odd positive integer } i \text { such that } d \mid\left(p^{\nu i}+1\right), \\ 0 & \text { otherwise. }\end{cases}
$$

The following lemma is a straightforward generalization of the case where $p=2$ (see [13, Lemma 3.5].)

Lemma 5.4. Let $p$ be a prime and let $\nu$ be a positive integer. Let $A$ be a finite abelian group such that $p \nmid|A|$ and let $a \in A$. Then $S_{p^{\nu}}(a)$ is of type $I^{\prime}$ if and only if $\lambda\left(\operatorname{ord}(a), p^{\nu}\right)=1$.

Proposition 5.5. Let $p$ be a prime and let $\nu$ be a positive integer. Let $A$ be a finite abelian group of exponent $N$. If $p \nmid N$, then

$$
r_{I^{\prime}}+r_{I^{\prime}}=\sum_{d \mid N} \lambda\left(d, p^{\nu}\right) \frac{\mathcal{N}_{A}(d)}{\operatorname{ord}_{d}\left(p^{\nu}\right)}+\frac{1}{2} \sum_{d \mid N}\left(1-\lambda\left(d, p^{\nu}\right)\right) \frac{\mathcal{N}_{A}(d)}{\operatorname{ord}_{d}\left(p^{\nu}\right)}
$$

where $\mathcal{N}_{A}(d)$ denotes the number of elements of order $d$ in $A$ determined in [1].

Proof. Using the argument similar to those in [13, Remark 2.5], for each $d \mid N$, the elements of order $d$ in $A$ are partitioned into $p^{2 \nu}$-cyclotomic classes of the same size $\frac{\mathcal{N}_{A}(d)}{\operatorname{ord}_{d}\left(p^{2 \nu}\right)}$. By Lemma [5.4, it follows that

$$
r_{I^{\prime}}=\sum_{d \mid N} \lambda\left(d, p^{\nu}\right) \frac{\mathcal{N}_{A}(d)}{\operatorname{ord}_{d}\left(p^{\nu}\right)} \text { and } r_{I^{\prime}}=\frac{1}{2} \sum_{d \mid N}\left(1-\lambda\left(d, p^{\nu}\right)\right) \frac{\mathcal{N}_{A}(d)}{\operatorname{ord}_{d}\left(p^{\nu}\right)} .
$$

Hence, the result follows.

In the case where $A$ is a cyclic group of order $n$ with $p \nmid n$, the exponent of $A$ is $n$ and $\mathcal{N}_{A}(d)=\Phi(d)$ for all divisors $d$ of $n$, where $\Phi(d)$ denotes the Euler totient phi function. The next corollary follows. 
Corollary 5.6. Let $p$ be a prime and let $\nu$ be a positive integer. Let $n$ be a positive integer such that $p \nmid n$. Then the number of Hermitian complementary dual cyclic codes of length $n p^{k}$ over $\mathbb{F}_{p^{2 \nu}}$ equals the number of Hermitian complementary dual cyclic codes of length $n$ over $\mathbb{F}_{p^{2 \nu}}$ for all integers $k \geq 0$ which is equal to

$$
2^{\sum_{d \mid n} \lambda\left(d, p^{\nu}\right) \frac{\Phi(d)}{\operatorname{ord}_{d}\left(p^{\nu}\right)}+\frac{1}{2} \sum_{d \mid n}\left(1-\lambda\left(d, p^{\nu}\right)\right) \frac{\Phi(d)}{\operatorname{ord}_{d}\left(p^{\nu}\right)}} .
$$

From Proposition 5.5, for an arbitrary finite abelian group $A$ with $p \nmid|A|$, we have $r_{I^{\prime}}+r_{I^{\prime}} \geq 1$. Hence, there exist at least two Hermitian complementary dual abelian codes in $\mathbb{F}_{p^{\nu}}[A]$.

In Table 2, the number $r_{I^{\prime}}+r_{I^{\prime}}$ for group algebras $\mathbb{F}_{4}[A]$ is given for finite abelian groups $A$ of odd order less than 50 .

\begin{tabular}{|r|r|r|}
\hline Order of $A$ & \multicolumn{1}{|r|}{$A$} & $r_{I^{\prime}}+r_{I^{\prime}}$ \\
\hline 3 & $\mathbb{Z}_{3}$ & 3 \\
5 & $\mathbb{Z}_{5}$ & 2 \\
7 & $\mathbb{Z}_{7}$ & 2 \\
9 & $\mathbb{Z}_{3} \times \mathbb{Z}_{3}$ & 9 \\
& $\mathbb{Z}_{3^{2}}$ & 5 \\
11 & $\mathbb{Z}_{11}$ & 3 \\
13 & $\mathbb{Z}_{13}$ & 2 \\
15 & $\mathbb{Z}_{5} \times \mathbb{Z}_{3}$ & 6 \\
17 & $\mathbb{Z}_{17}$ & 3 \\
19 & $\mathbb{Z}_{19}$ & 3 \\
21 & $\mathbb{Z}_{7} \times \mathbb{Z}_{3}$ & 6 \\
23 & $\mathbb{Z}_{23}$ & 2 \\
25 & $\mathbb{Z}_{5} \times \mathbb{Z}_{5}$ & 7 \\
& $\mathbb{Z}_{5^{2}}$ & 3 \\
27 & $\mathbb{Z}_{3} \times \mathbb{Z}_{3} \times \mathbb{Z}_{3}$ & 27 \\
& $\mathbb{Z}_{3^{2}} \times \mathbb{Z}_{3}$ & 15 \\
& $\mathbb{Z}_{3^{3}}$ & 7 \\
29 & $\mathbb{Z}_{29}$ & 2 \\
31 & $\mathbb{Z}_{31}$ & 4 \\
33 & $\mathbb{Z}_{11} \times \mathbb{Z}_{3}$ & 9 \\
35 & $\mathbb{Z}_{7} \times \mathbb{Z}_{5}$ & 5 \\
37 & $\mathbb{Z}_{37}$ & 2 \\
39 & $\mathbb{Z}_{13} \times \mathbb{Z}_{3}$ & 6 \\
41 & $\mathbb{Z}_{41}$ & 3 \\
43 & $\mathbb{Z}_{43}$ & 7 \\
45 & $\mathbb{Z}_{5} \times \mathbb{Z}_{3} \times \mathbb{Z}_{3}$ & 18 \\
47 & $\mathbb{Z}_{5} \times \mathbb{Z}_{3^{2}}$ & 9 \\
49 & $\mathbb{Z}_{47}$ & 2 \\
& $\mathbb{Z}_{7} \times \mathbb{Z}_{7}$ & 8 \\
& $\mathbb{Z}_{7^{2}}$ & 3 \\
\hline
\end{tabular}

Table 2: The number $r_{I^{\prime}}+r_{I^{\prime}}$ for group algebras $\mathbb{F}_{4}[A]$

From the properties of elements in $A=\left(\mathbb{Z}_{2^{k}}\right)^{s}$ discussed in [19, Sections 2 and 3], a simplified formula for (12) is given for some families of finite abelian groups. 
Example 5.7. Let $p$ be an odd prime and let $A=\left(\mathbb{Z}_{2^{k}}\right)^{s}$ for some positive integers $s$ and $k$. Let $\gamma$ be the largest integer $0 \leq \gamma \leq k$ such that $2^{\gamma} \mid\left(p^{\nu}+1\right)$. From [19, Remark 2.7], we have

$$
r_{I^{\prime}}=2^{\gamma s} \text { and } r_{I^{\prime}}=\sum_{i=\gamma+1}^{k} \frac{2^{i s}-2^{(i-1) s}}{\operatorname{2ord}_{2^{i}}\left(p^{2 \nu}\right)} .
$$

Then $r_{I^{\prime}}+r_{I^{\prime}}=2^{\gamma s}+\sum_{i=\gamma+1}^{k} \frac{2^{i s}-2^{(i-1) s}}{2 \operatorname{ord}_{2^{i}}\left(p^{2 \nu}\right)}$, and hence, the number of Hermitian complementary dual abelian codes in $\mathbb{F}_{p^{2 \nu}}\left[\left(\mathbb{Z}_{2^{k}}\right)^{s}\right]$ is

$$
2^{2^{\gamma s}+\sum_{i=\gamma+1}^{k} \frac{2^{i s}-2^{(i-1) s}}{2 \operatorname{ord}_{2}\left(p^{2 \nu}\right)}} .
$$

Example 5.8. Let $p$ be a prime and let $q$ be an odd prime such that $\operatorname{gcd}(q, p)=1$. Let $A=\left(\mathbb{Z}_{q^{k}}\right)^{s}$ for some positive integers $s$ and $k$. From [19, Corollary 3.12], we have the following two cases.

Case I: There exists $a \in A \backslash\{0\}$ such that $S_{p^{\nu}}(h)$ is of type $I^{\prime}$. By [19, Corollary 3.12], we have that that $S_{p^{2 \nu}}(a)$ is of type $I^{\prime}$ for all $a \in A$ and

$$
r_{I^{\prime}}=1+\sum_{i=1}^{k} \frac{q^{i s}-q^{(i-1) s}}{\operatorname{ord}_{q^{i}}\left(p^{2 \nu}\right)} \text { and } r_{I^{\prime}}=0
$$

In this case, we have $r_{I^{\prime}}+r_{I^{\prime}}=r_{I^{\prime}}$, and hence, the number of Hermitian complementary dual abelian codes in $\mathbb{F}_{p^{2 \nu}}\left[\left(\mathbb{Z}_{q^{k}}\right)^{s}\right]$ is

$$
2^{1+\sum_{i=1}^{k} \frac{q^{i s}-q^{(i-1) s}}{\operatorname{ord}_{q^{i}\left(p^{2 \nu}\right)}}}
$$

Case II: There exists $a \in A \backslash\{0\}$ such that $S_{p^{2 \nu}}(a)$ is of type $I^{\prime}$. By [19, Corollary 3.12] , we have that $S_{p^{2 \nu}}(a)$ is of type $I I^{\prime}$ for all $a \in A \backslash\{0\}$, and hence,

$$
r_{I^{\prime}}=1 \text { and } r_{I^{\prime}}=\sum_{i=1}^{k} \frac{q^{i s}-q^{(i-1) s}}{\operatorname{2ord}_{q^{i}}\left(p^{2 \nu}\right)} .
$$

In this case, we have

$$
r_{I^{\prime}}+r_{I^{\prime}}=1+\sum_{i=1}^{k} \frac{q^{i s}-q^{(i-1) s}}{\operatorname{ord}_{q^{i}}\left(p^{2 \nu}\right)},
$$

and hence, the number of Hermitian complementary dual abelian codes in $\mathbb{F}_{p^{2 \nu}}\left[\left(\mathbb{Z}_{q^{k}}\right)^{s}\right]$ is

$$
2^{1+\sum_{i=1}^{k} \frac{q^{i s}-q^{(i-1) s}}{2 \operatorname{ord}_{q^{i\left(p^{2 \nu}\right)}}}} .
$$

\section{Conclusion and Remarks}

In this paper, a family of abelian codes with complementary dual in group algebras $\mathbb{F}_{p^{\nu}}[G]$ has been investigated under both the Euclidean and Hermitian inner products. The characterization of such codes have been given. Subsequently, the number of complementary dual abelian codes in $\mathbb{F}_{p^{\nu}}[G]$ has been shown to be independent of the Sylow $p$-subgroup of $G$ and it has been completely determined for every finite 
abelian group $G$, prime $p$, and positive integer $\nu$. A simplified formula for the enumeration has been provided in the case where $G$ is a cyclic group or a $q$-group with $p \neq q$.

For application purpose, it is of natural interest to continue the study on the efficiency of codes in this family. Hence, the determination of their minimum distances is an interesting problem as well.

\section{References}

[1] Benson, S.: Students ask the darnedest things: A result in elementary group theory. Math. Mag. 70, 207-211 (1997).

[2] Berman, S. D.: Semi-simple cyclic and abelian codes. Kibernetika 3, 21-30 (1967).

[3] Bernal, J. J., Simón, J. J.: Information sets from defining sets in abelian codes. IEEE Trans. Inform. Theory 57, 7990-7999 (2011).

[4] Carlet, C., Guilley, S.: Complementary dual codes for countermeasures to sidechannel attacks. Coding Theory and Applications 3, 97-105 (2015).

[5] Carlet, C., Daif, A., Danger, J.L., Guilley, S., Najm, Z., Ngo, X.T., Portebouef, T., Tavernier, C.: Optimized linear complementary codes implementation for hardware trojan prevention. In: Proceedings of European Conference on Circuit Theory and Design, 2015 August 24-26; Trondheim, Norway. Piscataway, USA: IEEE (2015).

[6] Chabanne, H.: Permutation decoding of abelian codes. IEEE Trans. Inform. Theory 38, 1826-1829 (1992).

[7] Ding, C., Kohel, D. R., Ling, S.: Split group codes. IEEE Trans. Inform. Theory 46, 485-495 (2000).

[8] Etesami, J., Hu, F., Henkel, W.: LCD codes and iterative decoding by projections, a first step towards an intuitive description of iterative decoding. In: Proceedings of IEEE Globecom, 2011 December 5-9 ; Texas, USA. Piscataway, USA: IEEE (2011).

[9] Guenda, K., Jitman, S., Gulliver, T. A.: Constructions of good entanglementassisted quantum error correcting codes. Des., Codes and Cryptogr., doi:10.1007/s10623-017-0330-z.

[10] Ishai, Y., Sahai, A., Wagner, D.: Private circuits: securing hardware against probing attacks. In: CRYPTO, vol. 2729 of Lecture Notes in Computer Science, pages 463-481. Springer, August 1721 2003. Santa Barbara, CA, USA.

[11] Jitman., S, Ling, S.: Quasi-abelian codes. Designs, Codes and Cryptography 74, 511-531 (2015).

[12] Jitman, S., Ling, S., Liu, H., Xie, X.: Abelian codes in principal ideal group algebras. IEEE Trans. Inform. Theory 59, 3046-3058 (2013). 
[13] Jitman, S., Ling, S., Solé, P.: Hermitian self-dual Abelian codes. IEEE Trans. Inform. Theory 60, 1496-1507 (2014).

[14] Massey, J.L.: Linear codes with complementary duals. Discrete Mathematics 106/107 337-342 (1992).

[15] Moree, P.: On the divisors of $a^{k}+b^{k}$. Acta Arithmetica LXXX, 197-212 (1997).

[16] Ngo, X.T., Guilley, S., Bhasin, S., Danger, J.L., Najm, Z.: Encoding the state of integrated circuits: a proactive and reactive protection against hardware trojans horses. In: Proceedings of WESS '14, 2014 October 12-17; New Delhi, India. New York, ACM (2014).

[17] Ngo, X.T., Bhasin, S., Danger, J.L., Guilley, S., and Najm, Z.: Linear complementary dual code improvement to strengthen encoded cirucit against Hardware Trojan Horses. In: Proceedings of IEEE International Symposium on Hardware Oriented Security and Trust (HOST): 2015 May 2015; Washington DC Metropolitan Area, USA. Piscataway, USA: IEEE (2015).

[18] Nicholson, W. K.: Local group rings. Canad. Math. Bull. 15, 137-138 (1972).

[19] Palines, H. S., Jitman, S., Dela Cruz, R. B.: Hermitian self-dual quasi-abelian codes. Journal of Algebra Combinatorics Discrete Structures and Applications, to appear.

[20] Rajan, B. S. , Siddiqi, M. U.: Transform domain characterization of abelian codes. IEEE Trans. Inform. Theory 38, 1817-1821 (1992).

[21] Sabin, R. E.: On determining all codes in semi-simple group rings. Lecture Notes in Comput. Sci. 673, 279-290 (1993).

[22] Sendrier, N.: Linear codes with complementary duals meet the GilberVarshamov bound. Discrete Math 285, 345-347 (2004).

[23] Yang, X., Massey, J.L.: The condition for a cyclic code to have a complementary dual. Discrete Mathematics 126, 391-393 (1994). 